Produto \& Produção, vol. 5, n. 3, p. 48-60, out. 2001

$\mathbf{P P}$

\title{
Contribuições da análise multicritério à obtenção de graus de proxi- midade no projeto de arranjos físicos
}

\author{
William David Morán Herrera, M.Sc. \\ Laboratório de Engenharia de Produção/CCT/UENF - Av. Alberto Lamego, 2000 - Campos/RJ - CEP:28.030-480 - \\ Fax(22) 27263730 - Fone (22) 27261632 - E-mail : willy@uenf.br
}

Helder Gomes Costa, D.Sc.

Laboratório de Engenharia de Produção/CCT/UENF - Av. Alberto Lamego, 2000 - Campos/RJ - CEP:28.030-480 Fax (22) 27263730 - Fone (22) 27261632 - E-mail : hgc@uenf.br

Departamento de Engenharia Mecânica - Escola de Engenharia - CTC/UFF - Niterói - RJ

Fax (21) 27174446 - Fone (21) 26207070 R.832 - E-mail : temhgc@vm.uff.br

Este trabalho propõe uma abordagem multicritério para a classificação e obtenção de graus de proximidade (GP) utilizados na construção de arranjos físicos. Os GP são valores que expressam a intensidade com que se deve priorizar a aproximação de duas áreas ou estações de trabalho em um arranjo físico, fornecendo subsídios aos analistas na elaboração destes arranjos. Esta abordagem fundamenta-se no emprego do ELECTRE TRI na modelagem deste problema, incorporando o tratamento científico da subjetividade inerente à resolução deste problema e permitindo considerar simultaneamente múltiplos critérios, mesmos que estes sejam conflitantes. Outra característica importante adicionada pela metodologia é a possibilidade de identificar incomparabilidades associadas à incoerências da modelagem.

Palavras-chave: graus de proximidade; arranjos físicos; electre tri.

This work considers a multicriteria approach for the sorting and obtein of clossenes degrees (GP) used in the construction of layouts. The GP are values that express the intensity with that must prioritize the aproximation of two areas or stations of work in a layout, supplying to aid the analysts in the elaboration of this one. This approach is based in ELECTRE TRI's employment in the modeling of this problem, incorporating the scientific treatment of the inherent subjectivity to the resolution of this problem and allowing consider multiple approaches simultaneously, same that these are conflicting. Another important feature added by the methodology is the possibility to identify to incomparability associates with the incoherence of the modeling.

Keywords: closeness degrees; layout; electre tri.

\section{Introdução}

Em um ambiente dinâmico, marcado por extrema competição seletiva e por altas velocidades de obsolescência, as organizações devem se tornar aptas a enfrentar as diversas transformações que ocorrem de forma acelerada. Neste contexto, Boljwin e Kumpe (1990) estabelecem que a competitividade está fundamentada em três pilares: produtividade, qualidade e inovação.

Parte significativa dos problemas que provocam uma baixa competitividade nas empresas, sobretudo nas empresas de pequeno e médio porte, podem ser atribuídos a fatores como: qualidade e confiabilidade baixas, custos altos, pouca flexibilidade, qualidade de vida dos trabalhadores e baixa disponibilidade. Os arranjos físicos ou layouts influenciam direta e indiretamente o desempenho da organização nestes contextos. Assim, uma das formas de obter maior competitividade é implantar projetos de arranjos físicos adequados (Talmasky, 1998). Portanto, encontrar novas propostas para a solução do problema de arranjos físicos apresenta-se como um relevante desafio no âmbito do desenvolvimento científico e tecnológico.

Existem duas abordagens principais para a determinação de layouts: a abordagem quantitativa; e, a abordagem qualitativa. A abordagem quantitativa é aquela que só utiliza critérios quantificáveis na determinação de layouts. A abordagem qualitativa é aquela que usa tanto critérios quantificáveis (objetivos) como critérios qualitativos (subjetivos). 
A complexidade para a solução de problemas de layout é amplificada em situações nas quais não se dispõe de uma base de dados confiável em relação aos fluxos de trabalho, custos, tempos de produção, dentre outros. Estes casos são cada vez mais comuns, pelo fato de se ter uma velocidade de obsolescência alta que induz a entrada de novos produtos ou processos de produção - para os quais não existe um histórico de produção. Segundo Francis (1974), para estes casos a abordagem qualitativa é especialmente recomendada. A abordagem qualitativa para problemas de arranjos físicos utiliza critérios de avaliação não mensuráveis juntamente com critérios mensuráveis. Geralmente, esta abordagem envolve o uso de julgamentos de valores emitidos por especialistas para a obtenção de graus de proximidade. Como exemplos de metodologias que utilizam graus de proximidade citam-se Seehof e Evans (1967), Muther (1978), Rosenblatt (1979), Dutta e Sahu (1982), Fortenberry e Fox (1985), Urban (1987), dentre outros.

$\mathrm{Na}$ abordagem qualitativa, um dos problemas que surge na determinação dos arranjos físicos é a obtenção de graus de proximidade entre as instalações. Apesar da solução desse problema envolver subjetividade associada aos julgamentos de valor, não se observa na literatura o uso de metodologias específicas desenvolvidas para lidar com este tipo de problema. Nesse sentido, dentre as diversas metodologias existentes destacam-se aquelas vinculadas a lógica nebulosa e ao Auxílio Multicritério à Decisão (AMD), desenvolvidas para lidar com problemas que apresentam critérios qualitativos e quantitativos.

\section{Objetivo}

As principais abordagens no desenvolvimento de layouts podem ser classificadas em qualitativa e quantitativa. Na abordagem qualitativa, são utilizados GP obtidos através de avaliações subjetivas, buscando reconhecer e incorporar a experiência e a subjetividade ao processo de construção de arranjos físicos.

Este trabalho tem por objetivo apresentar uma abordagem qualitativa que contribua à obtenção sistemática de Graus de Proximidade (GP), fundamentada nos conceitos do AMD - mais especificamente pelo emprego método ELECTRE TRI (EvaLuation Et Choice Traduisant la REalité). O presente artigo apresenta uma evolução do trabalho de Herrera e Costa (1998), incorporando os conceitos de Grau de Desejabilidade (GD) e de Grau de Indesejabilidade (GI) à obtenção dos Graus de Proximidade (GP).

\section{Metodologias usuais para o de- senvolvimento de aranjos físicos}

Nesta seção, apresenta-se uma breve descrição das principais metodologias identificadas na literatura para o desenvolvimento de arranjos físicos.

- Modelo ALDEP: Seehof e Evans (1967), propõem este modelo para a busca computacional de uma solução (muitas vezes não ótima) do problema do layout. O ALDEP (Automated Layout Design Program) consiste de um processo sistemático que busca maximizar o somatório dos GP entre os departamentos dispostos como vizinhos. No inicio o programa faz uma seleção aleatória de um dos departamentos e o coloca dentro de um layout de blocos (só considera blocos de dimensões iguais). Em seguida a matriz formada por todos os GP entre as instalações é revisada e o departamento que tem um GP maior é colocado dentro do layout de blocos. Este processo continua até que todos os departamentos sejam colocados ou não exista nenhum departamento disponível para colocação que tenha um GP grande. A pontuação final do layout é determinada pela totalidade de valores numéricos dos GP. O processo inteiro é repetido um número especificado de vezes, sendo importante mencionar que quanto maior o número de vezes que este processo for repetido, maior a chance da solução estar perto da ótima.

- Modelo SLP: Proposto por Muther (1978), o SLP (Systematic Layout Planning) usa os GP para determinar layouts "manualmente". Faz-se uma análise das inter-relações entre as áreas que buscam ser arranjadas à luz de um conjunto de critérios de avaliação, determinando-se a importância relativa das relações em função de uma "carta de interligações”, como ilustrada na Figura 1.

Um valor alto para o GP de duas áreas (parte superior de cada losango) significa que no layout de blocos estas áreas devem ser arranjadas próximas. Um valor baixo do grau de proximidade significará que as áreas precisam ficar arranjadas mais afastadas. Assim, da carta de interligações se obtém um layout de blocos final. Neste modelo os GP são estimados através de julgamentos de valor. Deve-se salientar que determinar um layout seguindo este modelo é um processo "manual", o que implica uma maior dificuldade quando há uma grande quantidade de áreas por arranjar.

- O Modelo de Rosenblat: Rosenblat (1979), desenvolveu um modelo "multi-goal", onde explora dois objetivos, o qualitativo e o quantitativo. Nele basicamente busca-se minimizar o custo de manipulação dos materiais e por outro lado maximizar o somatório dos GP. A formulação é a seguinte: 


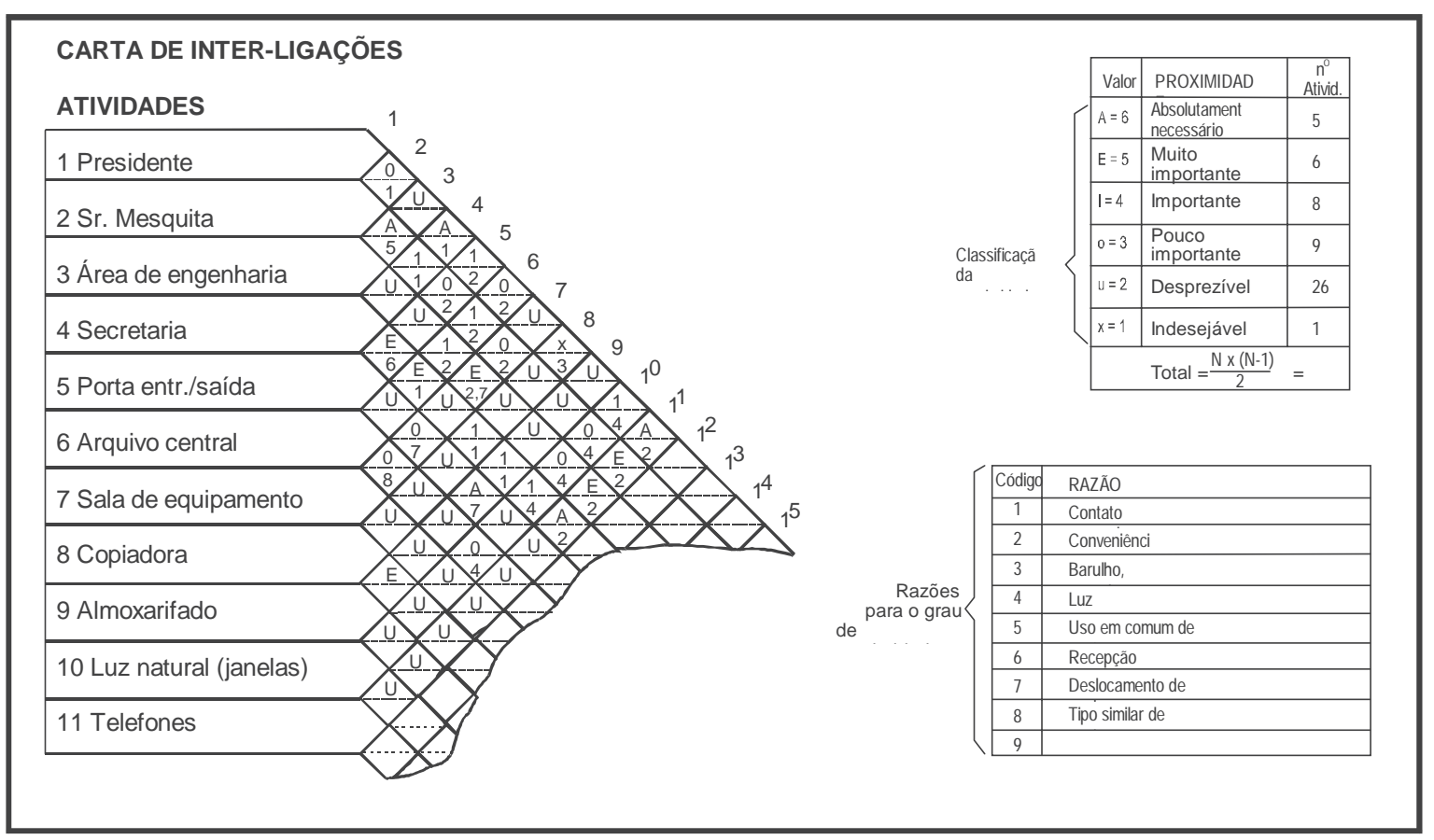

Figura 1. Exemplo de uma Carta de Interligações do SLP

$X_{\mathrm{ijj}}:=\left\{\begin{array}{l}1, \text { se o departamento "i”" é atribuído à localização “j”, } \\ 0 \text {, caso contrario }\end{array}\right.$

$a_{i j k l}=\left\{\begin{array}{lll}f_{i k} d_{j l}, & \text { se } i \neq k \text { ou } j \neq l, \\ f_{i i} d_{j j}+c_{i j}, & \text { se } i=k \text { e } j=1, \text { caso contrario. }\end{array}\right.$

$b_{i j k l}=\left\{\begin{array}{l}r_{\text {il }} \text { (grau de proximidade entre "i" e "l”), } \\ \text { se a localização "j” é adjacente da localização "k", } \\ 0 \text {, caso contrario. }\end{array}\right.$

$\mathrm{W}_{1}$ : é o peso da parte quantitativa da função.

$\mathrm{W}_{2}$ : é o peso da parte qualitativa da função.

Usa-se um intervalo de valores entre 6 e 1 para os GP, permitindo que o sistema de pontuação dos GP aumente quando os departamentos têm fronteiros comuns e ainda esquinas compartilhadas, além disso, usa como solução inicial layouts obtidos de métodos como o ALDEP, fazendo melhoras em função de análises gráficas dos mesmos.

- Modelo de Dutta e Sahu: Este modelo, proposto em Dutta e Sahu (1982), propõe uma abordagem similar ao modelo de Rosenblat, diferenciando-se por não permitir que o sistema de pontuação aumente quando dois departamentos têm esquinas compartilhadas.

- Modelo de Fortenberry e Cox: Neste modelo Fortenberry e Cox (1985) ressaltam que o uso de graus de proximidade num intervalo de valores entre 5 e -1, dedicando maior ênfase à separação entre áreas com graus de proximidade indesejáveis (negativos).

- Modelo de Urban: Assim como o modelo Fortenberry e Cox, o modelo proposto em Urban (1987) também usa um intervalo de valores entre 5 e -1 para os graus de proximidade, fornecendo uma boa separação entre departamentos com GP indesejáveis, particularmente aqueles com baixos fluxos de trabalho.

- Modelo de Evans, Wilhelm e Karwowski:

Considerando que muitos dos fatores presentes na determinação de layouts envolvem incertezas, Evans et al. (1987) propõem o uso de técnicas baseadas na fuzzy logic (lógica nebulosa). Basicamente define-se um conjunto de variáveis (proximidade e importância) e funções de pertinência para estabelecer que área deve entrar primeiro na construção de um layout de blocos.

- Modelo de Shang: Propõe-se 3 fases para lidar com o aspecto qualitativo na obtenção de layouts. Shang (1993) utiliza primeiramente o Analytic Hierarchy Process (AHP) para determinar os pesos dos critérios considerados, e numa segunda fase encontra as prioridades (graus de proximidade) para cada par das instalações consideradas para 
arranjar. Finalmente apresenta um "modelo aditivo" - parecido com o de Urban - que propõe a utilização de uma heurística baseada no simulated annealing para gerar uma solução.

- O modelo de Malakooti e Tsurushima: Considerando que a opinião dos especialistas ainda é muito importante na determinação de layouts, Malakooti e Tsurushima (1989) propõem um sistema especialista que estruturado em duas fases.

- Primeira fase: constrói-se um layout de blocos baseado num conjunto de regras e restrições.

- Segunda fase: desenvolvem-se melhorias ao layout mediante a interação com um decisor (especialista). O decisor usa uma escala do tipo A, E, I, O, U e X, para expressar suas prioridades em relação às regras e restrições.

Os modelos acima apresentados e de variações dos mesmos, observa-se a influencia e importância que os GP tem na concepção de layouts, podendo ser considerados como a base para gerar layouts de blocos. Observa-se, também, que em muitas situações os GP são emitidos por especialistas, com base em suas experiências e sem o emprego de uma metodologia mais refinada e eficaz, sobretudo quando o número de critérios é grande. Fica patente que a

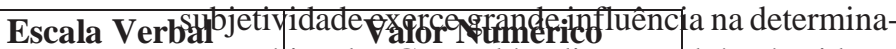
Extrema ção prática dos GP e, qléem disso, também é evidente

Alta que há carência de umz̧a abordagem científica que a

Média reconhø̧̧̧a e a trate dęidamente.

Baixa Desprezívef Metodologia proposta

A metodologia aqui proposta está estruturada no emprego da análise multicritério no auxílio à obtenção sistemática dos GP. Esta metodologia busca incorporar um tratamento científico mais refinado da subjetividade presente neste processo, visando uma melhor determinação dos arranjos físicos. Mais especificamente, se faz uso do método ELECTRE TRI e representa uma melhora ao trabalho apresentado em Herrera e Costa (1999). A Figura 2 ilustra as etapas desta metodologia, as quais estão descritas nos próximos tópicos.

\subsection{Caracterização do problema}

Nesta etapa, obtém-se os dados que caracterizam o problema e que auxiliarão na definição dos critérios de avaliação e a emissão de julgamentos de valor. Os dados são obtidos a partir da análise do produto, da análise do processo e do dimensionamento das áreas. Busca-se conhecer as características gerais do produto tais como tamanho, forma, peso e mercado alvo, dentre outros.

\subsection{Determinação dos critérios de avalia- ção e seus pesos (importância)}

Nesta fase um especialista (ou um grupo de especialistas) estabelece os critérios mais adequados de avaliação, baseado em seu conhecimento e perícia, além do peso de cada critério. Consideram-se dois tipos critérios, os de desejabilidade que expressam quanto é desejável dispor duas áreas próximas. Os critérios de indesejabilidade expressam o quanto é indesejável dispor duas áreas próximas.

Saaty (1999) sugere que sejam utilizadas escalas com "7 \pm 2 opções", isto é, uma escala com 5 ou 9 posições diferentes para a emissão dos julgamentos. Portanto, sabendo que a escala proposta em Muther (1978) -que serve de base na maioria dos modelos que usam GP - não fere a proposta de Saaty, e considerando os trabalhos de Freitas (1997), Freitas e Costa (1998), Herrera e Costa (1999), Santafé (1999) e Herrera (2000), que trabalham com questões semelhantes, utiliza-se à escala ilustrada na Tabela 1. É interessante ressaltar que Costa (2000), introduz a idéia do valor 0 (zero) associado a julgamentos desprezíveis. Este fato tem conseqüências interessantes sobre as classificações desenvolvidas pelo ELECTRE TRI, pois o valor 1 (um) associado a julgamentos desprezíveis pode trazer interpretações equivocadas quanto aos resultados obtidos pelo ELECTRE TRI.

\subsection{Determinação das categorias de acei- tação e de rejeição}

O ELECTRE TRI classifica alternativas em relação a um conjunto de categorias predefinidas, onde cada categoria é limitada por dois perfis adjacentes, como ilustrado na Figura 3. Além disso, cada perfil (determinado pelo especialista) estabelece limites diferentes para cada categoria. Assim, o perfil 1 é o limite superior da categoria 1 e que por sua vez é o limite inferior da categoria 2. No presente trabalho serão determinados uns conjuntos de categorias, tanto para os critérios de aceitação como para os de rejeição.

Tabela 1. Escala verbal para a determinação dos pesos dos critérios 


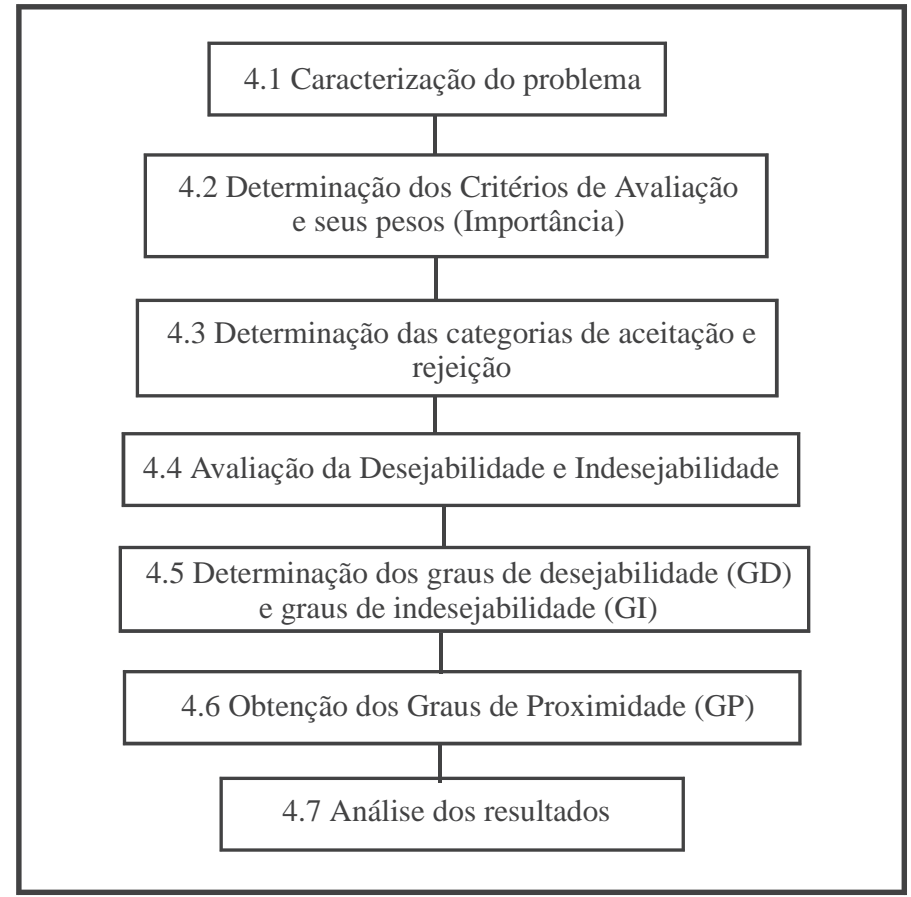

Figura 2. Metodologia Proposta

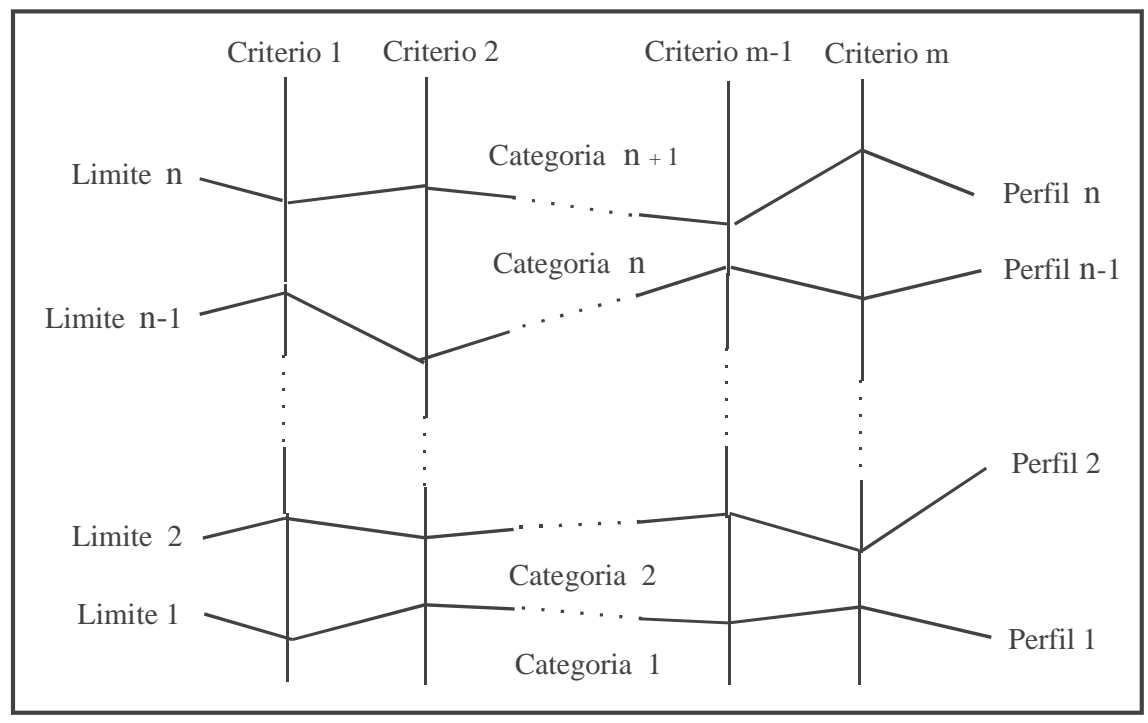

Figura 3: Classificação de alternativas segundo o Electre Tri

\subsection{Avaliação da desejabilidade e indesejabilidade}

Baseado em sua experiência, e nos valores nominais e numéricos das escalas utilizadas para os julgamentos (ilustradas nas Tabelas 2 e 3), o especialista emite:

- Julgamentos de Desejabilidade $\left(\mathrm{D}_{\mathrm{j}}(\mathrm{m}, \mathrm{n})\right)$ : Representam a intensidade com que se procura ou deseja aproximar duas áreas "m" e " $n$ ", segundo o critério de desejabilidade "j".
Tabela 2. Escala para os julgamentos da desejabilidade

\begin{tabular}{|c|c|}
\hline DESEJABILIDADE & Valor Numérico \\
Escala Verbal & $\mathbf{D}_{\mathbf{j}}$ \\
\hline Absoluta & 4 \\
Alta & 3 \\
Média & 2 \\
Baixa & 1 \\
Desprezível & 0 \\
\hline
\end{tabular}


- Julgamentos de Indesejabilidade $\left(\mathrm{I}_{\mathrm{j}}(\mathrm{m}, \mathrm{n})\right)$ : Representam a intensidade com que se procura afastar duas áreas "m" e "n", segundo o critério de indesejabilidade "j".

Tabela 3. Escala para os julgamentos da Indesejabilidade

\begin{tabular}{|c|c|}
\hline $\begin{array}{c}\text { INDESEJABILIDADE } \\
\text { Escala Verbal }\end{array}$ & $\begin{array}{c}\text { Valor Numérico } \\
\mathbf{I}_{\mathbf{j}}\end{array}$ \\
\hline Absoluta & -4 \\
Alta & -3 \\
Média & -2 \\
Baixa & -1 \\
Desprezível & 0 \\
\hline
\end{tabular}

\subsection{Determinação dos graus de desejabilidade e os graus de indesejabilidade}

Nesta fase busca-se determinar os graus de desejabilidade e os graus de indesejabilidade globais para cada par de áreas "m" e "n" $(G D(m, n)$ e GI(m.n) respectivamente).

Caso, em algum critério tenha-se $I_{j}(m, n)$ igual a “-4” (absolutamente indesejável), o GI(m,n) será igual a “4", indicando uma necessidade absoluta do afastamento das áreas $(\mathrm{m}, \mathrm{n})$. Caso isto não ocorra, utiliza-se o ELECTRE TRI para encontrar os GD(m,n) e GI(m,n) considerando os julgamentos $\operatorname{Dj}(m, n)$ e $\mathrm{Ij}(m, n)$ e os pesos de cada critério .

Além das categorias, perfis e limites definidos, de acordo com o ELECTRE TRI (ver anexo) é necessário estabelecer os seguintes parâmetros: limite de corte (1); limite de preferência (p); e limite de indiferença (q). No que diz respeito destes parâmetros, sugere-se utilizar valores de $1=0,76, p=0,1$ e $q=0,1$.

\subsection{Obtenção dos graus de proximidade (GP)}

Após a obtenção dos GD(m,n) e os GI(m,n), se procede à comparação dos mesmos para obter o grau de proximidade para as instalações "m" e " $n$ " $(\mathrm{GP}(\mathrm{m}, \mathrm{n}))$. O propósito desta comparação é categorizar os julgamentos e descobrir se existe uma maior preferência de dispor as áreas próximas (signo positivo), ou se existe uma maior preferência para que as áreas fiquem afastadas (signo negativo). Observase que $\mathrm{GP}(\mathrm{m}, \mathrm{n})$ só pode tomar algum valor inteiro no intervalo $[4,-4]$. O GP(m,n) pode ser definido da seguinte forma:

$\mathrm{GP}(\mathrm{m}, \mathrm{n})=\left\{\begin{array}{c}\mathrm{GI}(\mathrm{m}, \mathrm{n}), \text { se } \mathrm{GI}(\mathrm{m}, \mathrm{n})=-4 \\ \text { Caso contrario: } \\ \mathrm{GD}(\mathrm{m}, \mathrm{n}), \mathrm{se} \mathrm{GD}(\mathrm{m}, \mathrm{n})+\mathrm{GI}(\mathrm{m}, \mathrm{n}) \geq 0, \text { ou } \\ \mathrm{GI}(\mathrm{m}, \mathrm{n}), \text { se } \mathrm{GI}(\mathrm{m}, \mathrm{n})=\mathrm{GD}(\mathrm{m}, \mathrm{n})<0\end{array}\right.$
- GP(m,n) é o grau de proximidade das áreas “m” e "n".

- GI (m,n) é o grau de indesejabilidade das áreas "m" e "n",

- GD(m,n) é o grau de desejabilidade das áreas "m” e "n",

\subsection{Análise dos resultados}

Para fazer uma análise correta dos resultados, tem-se que entender primeiro como o ELECTRE TRI classifica as alternativas. O ELECTRE TRI tem dois procedimentos de classificação diferentes denominados otimista e pessimista respectivamente (ver Anexo).

Quando nos procedimentos otimista e pessimista uma alternativa é classificada na mesma categoria, o ELECTRE TRI classifica a alternativa naquela categoria. Quando os procedimentos otimista e pessimista classificam uma alternativa em categorias diferentes, é caracterizada uma incomparabilidade. Como o procedimento de classificação pessimista é mais rigoroso, neste trabalho sugere-se a adoção da classificação obtida no procedimento pessimista caso aconteça uma incomparabilidade.

As incomparabilidades podem surgir basicamente em dois casos, devido a uma modelagem errada do problema, ou por julgamentos inconsistentes do especialista que geram supervalorização ou subvalorização da alternativa na classificação final.

Portanto, neste problema, após a obtenção dos GP, tem-se que fazer uma análise das classificações obtidas para identificar incomparabilidades na obtenção dos graus de desejabilidade e indesejabilidade respectivamente e dessa forma detectar qualquer problema que possa surgir na classificação final. Deve-se lembrar que tanto a modelagem errada quanto os julgamentos inconsistentes acarretarão soluções de baixa qualidade.

\section{Exemplo de aplicação da metodologia proposta}

O exemplo mostra de forma generalizada como obter os GP para cinco áreas segundo a metodologia proposta, visando um caráter exclusivamente didático. Os julgamentos foram arbitrados de forma a destacar as características extremas da metodologia proposta.

\subsection{Caracterização do problema}

Tratando-se de um exemplo de aplicação que busca simular a metodologia proposta, o levantamento de dados e os cálculos efetuados nesta fase serão

Onde : 
omitidos para deixar o exemplo simplificado. Supõe-se que foram feitas todas as análises referidas a esta fase.

\subsection{Determinação dos critérios de avalia- ção e seus pesos (importância)}

De forma geral os critérios de desabilidade e indesejabilidade, e seus códigos são mostrados nas Tabelas 4 e 5 . Os pesos foram estimados segundo a escala apresentada na Tabela 1.

\subsection{Determinação das categorias de acei- tação e de rejeição}

As categorias de aceitação e rejeição neste exemplo são aqueles apresentados na Figura 4. Esta figura também ilustra os perfis que limitam estas categorias.

Tabela 5. Critérios de Indesejabilidade

\begin{tabular}{|l|c|c|}
\hline Critérios de Indesejabilidade & Pesos & Código \\
\hline Contaminação & 4 & $\mathrm{C}_{\mathrm{i} 1}$ \\
\hline Barulho & 2 & $\mathrm{C}_{\mathrm{i} 2}$ \\
\hline Temperatura & 1 & $\mathrm{C}_{\mathrm{i} 3}$ \\
\hline Poeira & 3 & $\mathrm{C}_{\mathrm{i} 4}$ \\
\hline
\end{tabular}

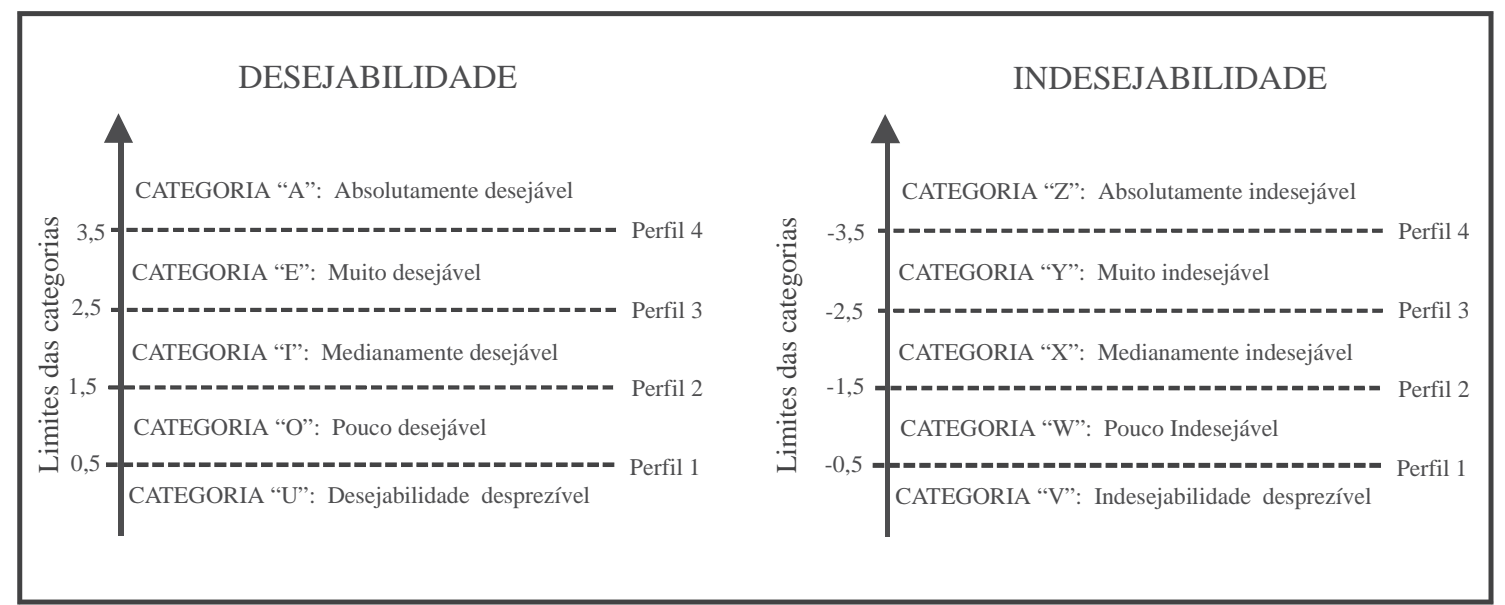

Figura 4: Categorias, perfis e limites dos critérios de desejabilidade e indesejabilidade

Tabela 6. Julgamentos de Desejabilidade e Indesejabilidade para todos os pares de áreas 


\subsection{Avaliação da desejabilidade e da indesejabilidade}

A desejabilidade e indesejabilidade de se aproximar duas áreas foi julgada em todos os critérios, com base nas escalas de julgamentos apresentadas nas Tabelas 2 e 3. Os resultados obtidos destes julgamentos estão ilustrados na Tabela 6, apresentada a seguir:

\subsection{Determinação dos graus de desejabilidade e os graus de indesejabilidade}

A partir dos julgamentos $\mathrm{D}_{\mathrm{j}}(\mathrm{m}, \mathrm{n})$ e $\mathrm{Ij}(\mathrm{m}, \mathrm{n})$, mostrados na Tabela 6, utilizou-se o método ELECTRE TRI para a classificação dos graus de desejabilidade $(\operatorname{GDj}(m, n))$ e de indesejabilidade e $\operatorname{GIj}(m, n)$.

Com base nos procedimentos apresentados na seção 4, foram obtidas três classificações: pessimista, otimista e final. As Tabelas 7 e 8 ilustram as classificações obtidas, adotando-se $1=0,76, \mathrm{p}=0,1$, e $\mathrm{q}=0,1$.

\subsection{Obtenção do grau de proximidade} (GP)

Com base nos valores obtidos nas Tabelas 7 e 8, foram determinados os $\mathrm{GP}(\mathrm{m}, \mathrm{n})$ a partir da adoção da regra proposta na seção 4.5, do presente artigo. Estes resultados estão ilustrados na Tabela 9.

\subsection{Análise dos resultados}

A Tabela 8 ilustra os graus de proximidade (GP) obtidos. Ao fazer a análise dos resultados, observa-se a presença de incomparabilidades na obtenção dos GP. Estas incomparabilidades são identificadas por diferenças entre as classificações otimista e pessimista, para o mesmo par de áreas $(\mathrm{m}, \mathrm{n})$ a ser arranjado. Neste exemplo específico, as incomparabilidades foram obtidas para as áreas $(1,4)$ e $(2,3)$, para as quais obtiveram-se GD $(1,4)$ e $\mathrm{GD}(2,3)$ diferentes nas classificações otimista e pessimista. Isto aconteceu devido a que se tem julgamentos com valores muito diferentes nos critérios, sendo que o caso mais grave é

Tabela 7. Classificação dos GD(m,n) e GI(m.n) segundo o Electre Tri

\begin{tabular}{|c|c|c|c|c|c|c|}
\cline { 2 - 7 } \multicolumn{1}{c|}{} & \multicolumn{6}{c|}{ Avaliação dos GD(m,n) } \\
\hline \multirow{2}{*}{$\begin{array}{c}\text { Áreas } \\
(\mathrm{m}, \mathrm{n})\end{array}$} & \multicolumn{2}{c}{ Pessimista } & \multicolumn{2}{c|}{ Otimista } & \multicolumn{2}{c|}{ Final } \\
\cline { 2 - 8 } & Num. & Verbal & Num. & Verbal & Num. & Verbal \\
\hline$(1,2)$ & 4 & A & 4 & A & 4 & A \\
\hline$(1,3)$ & 4 & A & 4 & A & 4 & A \\
\hline$(1,4)$ & 0 & U & 4 & A & 0 & U \\
\hline$(1,5)$ & 0 & U & 0 & U & 0 & U \\
\hline$(2,3)$ & 2 & I & 4 & A & 2 & I \\
\hline$(2,4)$ & 0 & U & 0 & U & 0 & U \\
\hline$(2,5)$ & 0 & $\mathrm{U}$ & 0 & $\mathrm{U}$ & 0 & $\mathrm{U}$ \\
\hline$(3,4)$ & 0 & $\mathrm{U}$ & 0 & $\mathrm{U}$ & 0 & $\mathrm{U}$ \\
\hline$(3,5)$ & 0 & $\mathrm{U}$ & 0 & $\mathrm{U}$ & 0 & $\mathrm{U}$ \\
\hline$(4,5)$ & 0 & $\mathrm{U}$ & 0 & $\mathrm{U}$ & 0 & $\mathrm{U}$ \\
\hline
\end{tabular}

Tabela 8. Classificação dos GD(m,n) e GI(m.n) segundo o Electre Tri

\begin{tabular}{|c|c|c|c|c|c|c|}
\hline \multirow{3}{*}{$\begin{array}{c}\text { Áreas } \\
(\mathrm{m}, \mathrm{n})\end{array}$} & \multicolumn{6}{|c|}{ Avaliação dos GI(m,n) } \\
\hline & \multicolumn{2}{|c|}{ Pessimista } & \multicolumn{2}{|c|}{ Otimista } & \multicolumn{2}{|c|}{ Final } \\
\hline & Num. & Verbal & Num. & Verbal & Num. & Verbal \\
\hline$(1,2)$ & 0 & $\mathrm{~V}$ & 0 & $\mathrm{~V}$ & 0 & $\mathrm{~V}$ \\
\hline$(1,3)$ & 0 & $\mathrm{~V}$ & 0 & $\mathrm{~V}$ & 0 & $\mathrm{~V}$ \\
\hline$(1,4)$ & -2 & $X$ & -2 & $X$ & -2 & $X$ \\
\hline$(1,5)$ & 0 & $\mathrm{~V}$ & 0 & $\mathrm{~V}$ & 0 & $\mathrm{~V}$ \\
\hline$(2,3)$ & 0 & $\mathrm{~V}$ & 0 & $\mathrm{~V}$ & 0 & $\mathrm{~V}$ \\
\hline$(2,4)$ & 0 & $\mathrm{~V}$ & 0 & $\mathrm{~V}$ & 0 & $\mathrm{~V}$ \\
\hline$(2,5)$ & 0 & $\mathrm{~V}$ & 0 & $\mathrm{~V}$ & 0 & $\mathrm{~V}$ \\
\hline$(3,4)$ & 0 & $\mathrm{~V}$ & 0 & $\mathrm{~V}$ & 0 & $\mathrm{~V}$ \\
\hline$(3,5) *$ & & & & & $-4 *$ & $\mathrm{Z}$ \\
\hline$(4,5) *$ & & & & & $-4 *$ & Z \\
\hline
\end{tabular}


Tabela 9. Classificação dos Graus de Proximidade

\begin{tabular}{|c|c|c|}
\hline $\begin{array}{c}\text { Áreas } \\
(\mathrm{m}, \mathrm{n})\end{array}$ & $\begin{array}{c}\text { Valor Numérico } \\
\text { dos GP(m,n) }\end{array}$ & $\begin{array}{c}\text { Classificação } \\
\text { dos GP(m,n) }\end{array}$ \\
\hline$(1,2)$ & 4 & A: Absolutamente desejável \\
\hline$(1,3)$ & 4 & A: Absolutamente desejável \\
\hline$(1,4)$ & -2 & X: Medianamente indesejável \\
\hline$(1,5)$ & 0 & U: Desejabilidade desprezível \\
\hline$(2,3)$ & 2 & I: Medianamente desejável \\
\hline$(2,4)$ & 0 & U: Desejabilidade desprezível \\
\hline$(2,5)$ & 0 & U: Desejabilidade desprezível \\
\hline$(3,4)$ & 0 & U: Desejabilidade desprezível \\
\hline$(3,5)$ & -4 & Z: Absolutamente indesejável \\
\hline$(4,5)$ & -4 & Z: Absolutamente indesejável \\
\hline
\end{tabular}

o de $\mathrm{GD}(1,4)$ onde as classificações otimista e pessimista foram A e U respectivamente, isto é, a maior e menor classificação, sugerindo uma falta de coerência no processo de modelagem e solução do problema. Observa-se a importância da identificação de incomparabilidades, que permite ao especialista/ decisor focar o problema com maior dedicação e cuidado -caso queira rever seus julgamentos-, ou ainda decidir-se por uma das classificações (pessimista ou otimista) que esteja mais próxima de seu parecer.

Como foi estabelecido, neste trabalho opto-se pela classificação pessimista caso aconteça uma incomparabilidade, mais esta é uma opção e não uma regra. Em casos mais extremos, esta incomparabilidade poderia até indicar rever os parâmetros da modelagem, e desta forma obter classificações mais de acordo com a percepção do especialista/decisor.

\section{Conclusões}

No presente trabalho propõe-se uma inédita metodologia para a obtenção dos GP necessários à determinação dos arranjos físicos. Não se revelou nenhuma ocorrência que indique algum impedimento na utilização da metodologia proposta, conseqüentemente, esta representaria uma alternativa na determinação de GP.

Como pontos destacáveis da metodologia proposta, citam-se:

- Reconhecimento de variáveis subjetivas no problema de arranjos físicos e o emprego de abordagens indicadas ao tratamento deste tipo de variáveis.

- Reconhecimento de múltiplos critérios no problema e o emprego de abordagens indicadas ao tratamento de problemas desta categoria.

- O caráter inovador como é tratado o conceito de indesejabilidade.
Esta metodologia é especialmente indicada para situações onde haja carência de dados quantificáveis. Em geral, esta situação ocorre nos seguintes contextos:

- Quando se têm critérios qualitativos.

- Quando, apesar de se utilizar critérios quantitativos, não se dispõe de uma base de dados confiável, ou os custos associados à obtenção da mesma a tornam proibitiva - situação que deve ser considerada, principalmente em pequenas e médias empresas.

- Quando do lançamento de novos produtos para os quais não exista um histórico bem definido.

Situação cada vez mais comum em um ambiente de inovação, como o que está sendo experimentado pela atual sociedade.

- Com relação aos resultados obtidos, estes não indicam restrições e mostram que é viável determinar taxas de proximidade segundo a metodologia proposta.

Outrossim, o uso dos GP na determinação de arranjos físicos é feito de maneira genérica. Isto é, são usados para qualquer tipo de instalação, seja de produção ou de serviços, embora alguns autores como Francis (1974) achem mais adequadas estas técnicas na determinação de layouts para empresas de serviços, onde a importância das interações clientes/instalações é maior e predominantemente do tipo qualitativo.

Em relação às desvantagens da metodologia, pode-se citar como principal, a emissão de uma maior quantidade de julgamentos. Esta quantidade é função do número de áreas ou estações de trabalho a dispor, e do número de critérios considerados. Evidentemente, ao se empregar uma análise multicritério, aumenta-se o número de julgamentos, quando comparada a uma análise monocritério, embora que este aumento seja menor do que o que seria obtido, ao se utilizar outras abordagens multicritério, como por exemplo, àquelas 
que empregam julgamentos paritários - como no caso do AHP.

Nesse sentido, considerando que do ponto de vista prático não são emitidos julgamentos para os pares de áreas que apresentam relacionamentos desprezíveis, e que muitas das vezes essa quantidade de julgamentos chega ao redor de $50 \%$ do total de julgamentos, fica claro que o número de julgamentos pode diminuir consideravelmente. Finalmente, é importante destacar a boa escolha do especialista, porque é ele quem por médio de sua perícia, experiência, e conhecimento, emitira os julgamentos necessários para a obtenção dos GP e depois validará os resultados.

\section{Referências bibliográficas}

BOLJWIN, P.; KUMPE, T. Manufacturing in the 1990's. Productivity, Flexibility and Innovation, Longe Range Planning, vol. 23, n 4, p. 44-57, 1990.

COSTA, H. G. Proposta para avaliação do grau de competitividade por ponderação de múltiplos critérios , Boletim Técnico Interno. Campos dos Goytacazes, RJ, Brasil: Laboratório de Engenharia de Produção/ CCT/UENF, 2000.

DUTTA, K.; SAHU, S. A multigoal heuristic for facilities design problems : MUGHAL. International Journal of Production Research, v. 20, n² 2, p. 147154, 1982.

EVANS, G.W.; WILHELM, M.R.; KARWOWSKI, W. A layout design heuristic employing the theory of fuzzy sets. International Journal of Production Research, vol. 25, n 10, p. 1431-1450, 1987.

FORTENBERRY, J.; COX, J. Multiple criteria approach to the facilities layout problem.

International Journal of Production Research, v. 23, $\mathrm{n}^{\circ} 4$, p. 777-782, 1985.

FRANCIS, R.; WHITE, J.A. Facility Layout and Location. Englewood Cliffs: Prentice - Hall, N.J., USA, 1974.

FREITAS, A.L.P. Emprego de uma abordagem multicritério na avaliação e classificação da qualidade de serviços, dissertação de Mestrado, LGEN/Setor de Engenharia de Produção, Universidade Estadual do Norte Fluminense, 1997.

FREITAS, A. L. P.; COSTA, H. Uma abordagem multicritério para avaliação e classificação da qualidade de serviços. In: XVII ENCONTRO NACIONAL DE ENGENHARIA DE PRODUÇÃO (ENEGEP 98), Gramado, Rio Grande do Sul, 1998. Anais ... CD Room.
HERRERA, William; COSTA, Helder. O método Electre III aplicado ao desenvolvimento de Arranjos Físicos. In: XIX ENCONTRO NACIONAL DE ENGENHARIA DE PRODUÇÃO (ENEGEP 99), Rio de Janeiro, 1999. Anais ... CD Room.

HERRERA, W. Uma abordagem multicritério no desenvolvimento de arranjos físicos, dissertação de Mestrado, LGEN/Setor de Engenharia de Produção, Universidade Estadual do Norte Fluminense, 2000.

MALAKOOTI, B.; TSURUSHIMA, A. An expert system using priorities for solving multiple-criteria facilities layout problems. International Journal of Production Research, vol. 27, n 5, p. 793-808, 1989.

MOSSEAU, V.; SLOWINSKI, R.; ZIELNIEWICCZ, P. Electre Tri 2.0a. Methodological Guide and User's Manual. Document Du Lamsade. Université Paris Dauphine, Fevreiro/1999.

MUTHER, R. Planejamento do layout: O sistema SLP. Editora Edgard Blücher, S.P., Brasil, 1978.

ROSENBLATT, M.J. The facilities layout problem: A multi-goal approach. International Journal of Production Research, vol. 17, no 4, p. 323-332, 1979.

SAATY, T.L. Decision Making for leaders: The Analytic Hierarchy Process for decision in a complex world. 3 ed. RWS Publications, Pittsburg, USA, 1999. $315 \mathrm{p}$.

SANTAFÉ, J.; COSTA, H.G. Metodologia para Análise de plantas industriais Sucro-alcooleiras utilizando Gerenciamento de Risco e Análise multicritério, dissertação de Mestrado, LGEN/Setor de Engenharia de Produção, Universidade Estadual do Norte Fluminense, 1999.

SEEHOF, J.M.; EVANS, W.O. Automated Layout Design Program. Journal of Industrial Engineering, vol. $18, \mathrm{n}^{\circ} 12$, p. 690-695, 1967.

SHANG, J.S. Multicriteria facility layout problem: An integrated approach. European Journal of Operational Research, vol. 66, p. 291-304, 1993.

TALMASKY, E.; DOS SANTOS, N. (1998). A ecoergonomia como auxiliar na concepção do espaço de trabalho. In: XVII ENCONTRO NACIONAL DE ENGENHARIA DE PRODUÇÃO (ENEGEP 98), Gramado, Rio Grande do Sul, 1998. Anais ... CD Room.

URBAN, T. A multiple criteria model for the Facilities Layout Problem. International Journal of Production Research, v. 25, no 12, p. 1805-1812, 1987. 
YU, W. Electre Tri - Aspects Methodologiques et Guide d'Utilization. Document du LAMSADE no 74. Université de Paris - Dauphine, Abril/1992.

\section{Agradecimentos}

Este trabalho foi viabilizado pelo apoio institucional da Universidade Federal Fluminense (UFF) e da Universidade Estadual do Norte Fluminense (UENF), além do apoio financeiro do Conselho Nacional de Desenvolvimento Científico e Tecnológico (CNPq), da Fundação Estadual do Norte Fluminense (FENORTE) e da Fundação Carlos Chagas de Amparo à Pesquisa do Estado do Rio de Janeiro (FAPERJ).

\section{Anexo: ELECTRE TRI}

Considerando a pouca divulgação do Método ELECTRE TRI na literatura técnica nacional, optouse por apresentar neste Anexo uma descrição deste. Maiores informações sobre o mesmo podem ser encontradas em Yu (1992) e Mousseau et al. (1999). O Método ELECTRE TRI pertence à família ELECTRE e caracteriza-se por tratar de problemas específicos de classificação ordenada. Ou seja: dado um conjunto de $\underline{\mathbf{A}}=\{\mathrm{a}, \mathrm{b}, \mathrm{c}, .$.$\} de alternativas,$ associa-as a um conjunto de categorias ou classes ordenadas $\underline{\mathbf{C}}=\left\{\mathrm{C}_{1}, \mathrm{C}_{2}, \ldots \ldots \mathrm{C}_{\mathrm{n}\}}\right.$, considerando o desempenho de $\underline{\mathbf{A}}$ a luz de um conjunto de critérios $\underline{\mathbf{F}}=\left\{\mathrm{g}_{1}, \mathrm{~g}_{2}, \ldots, \mathrm{g}_{\mathrm{m}}\right\}$. As classes são delimitadas por limites superiores e limites inferiores, conforme ilustrado na Figura A.1.

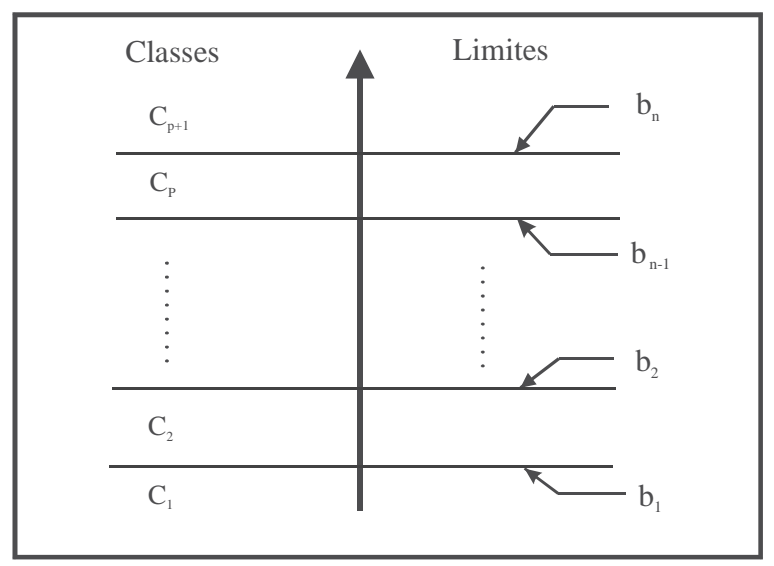

Figura A.1. Classes de equivalência no ELECTRE TRI

Este método integra funções específicas que dão suporte ao decisor no processo de preferência e reduzem o esforço cognitivo requerido na fase de modelagem. O ELECTRE TRI classifica as alternativas seguindo dois passos consecutivos: construção de uma relação de subordinação $S$, que caracteriza como as alternativas são comparadas aos limites das classes; e, exploração (através de procedimentos de classificação) da relação $S$.

\section{Relação de subordinação no ELECTRE TRI}

A relação de subordinação é construída para tornar possível a comparação de uma alternativa $a$ com um limite padrão $b_{h}$. A afirmação de que $a S b_{h}$, significa que " $a$ é ao menos tão boa quanto $b_{h}$ ". Na validação da afirmação $a S b_{h}$ (ou $b_{h} S a$ ), devem-se verificar duas condições:

- Concordância: para que $a S b_{h}$ (ou $b_{h} S a$ ) seja aceita, uma maioria suficiente de critérios deve ser a favor desta afirmação.

- Não-discordância: quando na condição de concordância esperada, nenhum dos critérios na minoria deve se opor à afirmação $a S b_{h}\left(\right.$ ou $\left.b_{h} S a\right)$.

Também devem ser considerados dois tipos de parâmetros associados aos de critérios intervém na construção de $S$ :

- O conjunto de coeficientes dos pesos ou importância $\left(k_{1}, k_{2}, \ldots, k_{m}\right)$, usado no teste de concordância quando se computa a importância relativa da união dos critérios que são a favor da afirmação $a S b_{h}$.

- O conjunto de limites de veto $\left(v_{1}\left(b_{h}\right), v_{2}\left(b_{h}\right), \ldots\right.$, $\left.v_{m}\left(b_{h}\right)\right), \forall h \quad B$, é usado no teste de discordância. $v_{j}\left(b_{h}\right)$ representa a menor diferença $g_{j}\left(b_{h}\right)-g_{j}(a)$ incompatível com a afirmação $a S b_{h}$.

Os seguintes passos são seguidos na obtenção desta relação:

- Computar o índice de concordância parcial $c_{j}\left(a, b_{h}\right)$ e $c_{j}\left(b_{h}, a\right)$,

- Computar o índice de concordância geral $c\left(a, b_{h}\right)$,

- Computar o índice de discordância parcial $d_{j}\left(a, b_{h}\right)$ e $d_{j}\left(b_{h}, a\right)$,

- Computar a relação de subordinação fuzzy conforme o índice de credibilidade $s\left(a, b_{h}\right)$,

- Determinar um corte $\lambda$ da relação fuzzy para obter uma relação de subordinação. Isto é: Se $\sigma\left(a, b_{h}\right) \quad \lambda \Rightarrow a S b_{h}$.

O índice de concordância parcial $c_{j}\left(a, b_{h}\right)$ expressa o quanto à afirmação " $a$ é ao menos tão boa quanto $b_{h}$ considerando o critério " $g$ ” é válida. Este índice é computado da seguinte forma:

- Quando $g_{j}$ tem uma direção de preferência crescente, $c_{j}\left(a, b_{h}\right)$ é computado como se segue: 


$$
\begin{aligned}
& -\operatorname{Se~} g_{j}(a) \leq g_{j}\left(b_{h}\right)-p_{j}\left(b_{h}\right) \text {, então } c_{j}\left(a, b_{h}\right)=0 \\
& -\operatorname{Se~} g_{j}\left(b_{h}\right)-p_{j}\left(b_{h}\right)<g_{j}(a) \leq g_{j}\left(b_{h}\right)-q_{j}\left(b_{h}\right), \quad \text { então } c_{j}\left(a, b_{h}\right)=\frac{\left[g_{j}(a)-g_{j}\left(b_{h}\right)+p_{j}\left(\frac{b_{h}}{2}\right)\right]}{\left[p_{j}\left(b_{h}\right)-q_{j}\left(b_{h}\right)\right]} \\
& -\operatorname{Se~} g_{j}\left(b_{h}\right)-q_{j}\left(b_{h}\right)<g_{j}(a), \text { então } c_{j}\left(a, b_{h}\right)=1
\end{aligned}
$$

- Quando $g_{j}$ tem uma direção de preferência decrescente, $c_{j}\left(a, b_{h}\right)$ é computado como se segue:

$$
\begin{aligned}
& -\operatorname{Se~} g_{j}(a) \geq g_{j}\left(b_{h}\right)+p_{j}\left(b_{h}\right), \text { então } c_{j}\left(a, b_{h}\right)=0 \\
& -\operatorname{Se~} g_{j}\left(b_{h}\right)+q_{j}\left(b_{h}\right) \leq g_{j}(a) \leq g_{j}\left(b_{h}\right)+p_{j}\left(b_{h}\right) \text {, então } c_{j}\left(a, b_{h}\right)=\frac{\left[g_{j}\left(b_{h}\right)-g_{j}(a)+p_{j}\left(b_{h}\right)\right]}{\left[p_{j}\left(b_{h}\right)-q_{j}\left(b_{h}\right)\right]} \\
& -\operatorname{Se~} g_{j}\left(b_{h}\right)+q_{j}\left(b_{h}\right)>g_{j}(a) \text {, então c c }\left(a, b_{h}\right)=1
\end{aligned}
$$

- O índice de concordância global $c_{j}\left(b_{h}, a\right)$ expressa até que ponto as avaliações de $a$ e $b_{h}$ em todos os critérios estão de acordo com a afirmação " $a$ subordina $b_{h}$ ":

$$
\mathrm{c}_{\mathrm{j}}\left(\mathrm{a}, \mathrm{b}_{\mathrm{h}}\right)=\quad \sum_{\mathrm{i} \in \mathrm{E}} \frac{\mathrm{k}_{\mathrm{j}}}{\mathrm{c}_{\mathrm{j}}\left(\mathrm{a}, \mathrm{b}_{\mathrm{h}}\right)} \underset{\mathrm{j} \in \mathrm{F}}{\sum_{\mathrm{j}}}
$$

- O índice de discordância parcial $d_{j}\left(a, b_{h}\right)$ expressa até que ponto o critério $g_{j}$ se opõe à afirmação " $a$ é ao menos tão boa quanto $b_{h}$ ", isto é, " $a$ subordina $b_{h}$ ". Um critério $g_{j}$ é considerado discordante com a afirmação " $a$ subordina $b_{h}$ " se, neste critério, $b_{h}$ é preferida à $a$. Neste caso de preferência crescente, o critério $g_{j}$ se opõe a um veto quando a diferença $g_{j}\left(b_{h}\right)-g_{j}(a)$ excede ao limite de veto $v_{j}\left(b_{h}\right)$.

- Quando $g_{j}$ tem uma direção de preferência crescente, $d_{j}\left(a, b_{h}\right)$ é computado como se segue:

- Se $g_{j}(a)>g_{j}\left(b_{h}\right)-p_{j}\left(b_{h}\right)$, então $d_{j}\left(a, b_{h}\right)=0$

- Se $g_{j}\left(b_{h}\right)-v_{j}\left(b_{h}\right)<g_{j}(a) \leq g_{j}\left(b_{h}\right)-p_{j}\left(b_{h}\right)$, então $d_{j}\left(a, b_{h}\right)=\frac{\left[g_{j}\left(b_{h}\right)-g_{j}(a)-p_{j}\left(b_{h}\right)\right]}{\left[v_{j}\left(b_{h}\right)-p_{j}\left(b_{h}\right)\right]}$ $\phi\left(a, b_{h}\right)=c\left(a, b_{h}\right) \prod_{j \in \in}-\operatorname{Fe}_{1} g_{j}\left(b_{j}\right)-{ }_{j}, b_{h}\left(b_{h}\right) \geq q_{j}(a)$, então $d_{j}\left(a, b_{h}\right)=1$

- Quahđo $c_{j}\left(a_{\text {teh }} h_{\text {h }}\right.$ uma direção de preferência decrescente, $d_{j}\left(a, b_{h}\right)$ é computado como se segue: $\overline{\mathrm{F}}=\left\{\mathrm{j} \in \mathrm{F} / \mathrm{d}_{\mathrm{i}}\left(\mathrm{a}, \mathrm{b}_{\mathrm{h}}\right)>\mathrm{c}\left(\mathrm{a}, \mathrm{b}_{\mathrm{h}}\right)\right\}$

$$
\begin{aligned}
& -\operatorname{Se~} g_{j}(a) \leq g_{j}\left(b_{h}\right)+p_{j}\left(b_{h}\right) \text {, então d }\left(a, b_{h}\right)=0 \\
& -\operatorname{Se~} g_{j}\left(b_{h}\right)+p_{j}\left(b_{h}\right)<g_{j}(a) \leq g_{j}\left(b_{h}\right)+v_{j}\left(b_{h}\right) \text {, então } d_{j}\left(a, b_{h}\right)=\underset{j}{\left[g_{j}(a)-g_{j}\left(b_{h}\right)-p_{j}\left(b_{h}\right)\right]}\left[v_{j}\left(b_{h}\right)-p_{j}\left(b_{h}\right)\right] \\
& -\operatorname{Se~} g_{j}\left(b_{h}\right)+v_{j}\left(b_{h}\right)<g_{j}(a) \text {, então } d_{j}\left(b_{h}, a\right)=1
\end{aligned}
$$

O grau de credibilidade da relação de subordinação $\sigma\left(a, b_{h}\right)$ expressa até que ponto " $a$ subordina $b_{h}$ " de acordo com o índice de concordância global $c_{j}\left(a, b_{h}\right) \mathrm{e}$ com o índice de discordância $d_{j}\left(a, b_{h}\right), \quad{ }_{j i \hat{F}}$ Calcula-se o índice de credibilidade $\sigma\left(a, b_{h}\right)$ e $\sigma\left(b_{h}, a\right)$ somandose os valores estabelecidos na relação de subordinação. O cálculo do índice de credibilidade $\sigma\left(a, b_{h}\right)$ é de acordo com os seguintes princípios:

Quando nenhum critério for discordante, a credibilidade da relação de subordinação $s\left(a, b_{h}\right)$ é igual ao índice de concordância $s\left(a, b_{h}\right)$.

- Quando um critério discordante se opõe ao veto para a afirmação " $a$ subordina $b_{h}$ " (i. é, $d_{j}\left(a, b_{h}\right)=$ $1)$, então o índice de credibilidade $\sigma\left(a, b_{h}\right)$ torna-se nulo (a afirmação " $a$ subordina $b$ " não é totalmente acreditável).

Quando um critério discordante é tal como $c\left(a, b_{h}\right)$ $<d_{j}\left(a, b_{h}\right)<1$, o índice de credibilidade $\sigma\left(a, b_{h}\right)$ torna-se mais baixo do que o índice de concordância $c\left(a, b_{h}\right)$, sendo justo o efeito de oposição deste critério.
A conclusão destes princípios é de que o índice de credibilidade $s\left(a, b_{h}\right)$ corresponde ao índice de concordância fraca por um eventual efeito de veto. Mais precisamente, o valor $s\left(a, b_{h}\right)$ é calculado como se segue $\left(s\left(b_{h}, a\right)\right.$ é calculado similarmente):

A tradução de uma relação de subordinação fuzzy obtida entre uma relação de subordinação $S$ é feita sobre o significado de um corte- $\lambda$, ( $\lambda$ é chamado de nível de corte). $\lambda$ é considerado como o menor valor do índice de credibilidade compatível com a afirmação de que " $a$ subordina $b$ ", i. é, $\sigma\left(a, b_{h}\right) \geq \lambda \Rightarrow a S b_{h}$. Assim, são definidas relações binárias de preferência $(>)$, indiferença (I) e incomparabilidade (R):

$$
\begin{aligned}
& \mathrm{aIb}_{\mathrm{h}} \Leftrightarrow \mathrm{aSb}_{\mathrm{h}} \text { é } \mathrm{b}_{\mathrm{h}} \mathrm{Sa} \\
& \mathrm{a}>\mathrm{b}_{\mathrm{h}} \Leftrightarrow a \mathrm{aSb}_{\mathrm{h}} \text { e não } \mathrm{b}_{\mathrm{h}} \mathrm{Sa} \\
& \mathrm{a}<\mathrm{b}_{\mathrm{h}} \Leftrightarrow \text { não } \mathrm{aSb}_{\mathrm{h}} \text { e } \mathrm{b}_{\mathrm{h}} \mathrm{Sa} \\
& \mathrm{aRb}_{\mathrm{h}} \Leftrightarrow \text { não } \mathrm{aSb}_{\mathrm{h}} \text { e não } \mathrm{b}_{\mathrm{h}} \mathrm{as}
\end{aligned}
$$




\section{Procedimentos de classificação}

A regra do procedimento de exploração é realizada para analisar o modo em que uma alternativa $a$ é comparada com os limites padrão determinados para a classe na qual $a$ deve ser enquadrada. Dois procedimentos de classificação são avaliados.

$\left.1^{\circ}\right) \mathrm{O}$ procedimento de classificação pessimista (ou conjuntivo) é descrito a seguir:

Comparar $a$ sucessivamente $\operatorname{com} b_{i}$, para $\mathrm{i}=\mathrm{p}, \mathrm{p}-$ $1, \ldots, 0$.

Que $b_{h}$ seja o primeiro limite padrão tal que $a S b_{h}$, classificando $a$ para a classe $C_{h+1}\left(\mathrm{a} \rightarrow \mathrm{C}_{\mathrm{h}+1}\right)$. Se $b_{h-1}$ e $b_{h}$ denotam o limite superior e inferior da classe $C_{h}$, o procedimento pessimista classifica a alternativa $a$ para a mais alta classe $C_{h}$ tal que $a$ subordine $b_{h-1}$, isto é, $a S b_{h-1}$. Quando se utiliza este procedimento com $\lambda=1$, uma alternativa $a$ pode ser enquadrada na classe $C_{h}$ somente se $g_{j}(a)$ for igual ou exceder $g_{j}\left(b_{h-I}\right)$ (pela soma dos limites) para cada critério (regra conjuntiva). Quando 1 decresce, o caráter conjuntivo desta regra é fraco.

$\left.2^{\circ}\right) \mathrm{O}$ procedimento de classificação otimista (ou disjuntivo) é descrito a seguir:

Comparar $a$ sucessivamente $\operatorname{com} b_{i}, \mathrm{i}=1,2, \ldots, \mathrm{p}$. Que $b_{h}$ seja o primeiro limite padrão tal que $b_{h}>a$, classificando $a$ na classe $C_{h}\left(\mathrm{a} \quad \mathrm{C}_{\mathrm{h}}\right)$.

$\mathrm{O}$ procedimento otimista (ou disjuntivo) classifica $a$ para a mais baixa classe $C_{h}$ para a qual o limite superior de $b_{h}$ é preferível a $a$, i. é, $b_{h}>a$. Quando se utiliza este procedimento $\operatorname{com} \lambda=1$, uma alternativa $a$ pode ser classificada na classe $C_{h}$ quando $g_{j}\left(b_{h}\right)$ exceder $g_{j}(a)$ (pela soma dos limites) ao menos para um critério (regra disjuntiva). Quando 1 decresce, o caráter disjuntivo desta regra é fraco.

$3^{\circ}$ ) Comparação entre os dois procedimentos de classificação:

Sendo estes dois procedimentos diferentes, conseqüentemente, pode ocorrer a classificação de algumas alternativas em diferentes classes. $\mathrm{O}$ exemplo seguinte explica, num nível teórico, a razão da possibilidade de divergência dos resultados de classificação.

Suponha-se que uma alternativa $a$ é classificada em $C_{i}$ e $C_{j}$ pelas regras de classificação pessimista e otimista, respectivamente. Espera-se:

- Que $C_{i}$ seja inferior ou igual à $C_{j}(i \leq j)$.

- Que $C_{i}$ seja inferior a $C_{j}$ quando $a$ é incomparável com todos os limites entre $C_{i}$ e $C_{j}\left(a R b_{t^{\prime}}\right.$ $f$, tal que $i<f j$ ). 